\title{
Li-Fi (Light Fidelity)
}

\author{
K. Lakshmisudha \\ Associate Professor \\ Dept. of Information \\ Technology, \\ SIES GST \\ $\operatorname{Nerul}(E)$.
}

\author{
Divya Nair \\ BE-IT \\ SIES GST \\ $\operatorname{Nerul}(\mathrm{E})$,
}

\author{
Aishwarya Nair \\ BE-IT \\ SIES GST \\ $\operatorname{Nerul}(\mathrm{E})$,
}

\author{
Pragya Garg \\ BE-IT \\ SIES GST, \\ $\operatorname{Nerul}(\mathrm{E})$,
}

\begin{abstract}
A person gets frustrated when he faces slow speed as many devices are connected to the same network. As the number of people accessing wireless internet increases, it's going to result in clogged airwaves. Li-Fi is transmission of data through illumination by taking the fiber out of fiber optics by sending data through a LED light bulb that varies in intensity faster than the human eye can follow.
\end{abstract}

As $\mathrm{Li}-\mathrm{Fi}$ is considered to be the optical version of $\mathrm{Wi}-\mathrm{Fi}$, some label it as fast and cheap wireless communication system. When LED is on digital 1 is transmitted and when it is off 0 is transmitted. Data can be encoded in the light by varying the flickering rate to generate strings of $1 \mathrm{~s}$ and $0 \mathrm{~s}$. The output appears to be constant to the human eye as the LED intensity is modulated rapidly.

This method of using rapid pulses of light to transmit information wirelessly is technically referred to as Visible Light Communication (VLC). Advancements promise a speed of $10 \mathrm{Gbps}$. Li-Fi can work even underwater.

If this technology can be put into practical use, every bulb can be used something like a Wi-Fi hotspot to transmit wireless data and will proceed toward a cleaner, greener, safer and brighter future. As the growing number of device accessing wireless internet are coming into use airwaves are becoming increasingly clogged making it more and more difficult to get a reliable, high speed signal.

\section{General Terms}

LED light source, VLC, Transmission

\section{Keywords}

Photodiode, Microcontroller

\section{INTRODUCTION}

$\mathrm{Li}-\mathrm{Fi}$ is the term used to label the wireless communication system through an optical source that makes the system fast and cheap. Li-Fi is based upon Visible Light Communication technology where data transmission is made through a light bulb whose intensity varies at a rate faster than the human eye can follow. The fiber out of the fiber optics is replaced by an LED source for the data transmission. The term Li-Fi was introduced by Harald Hass in his TED Global talk on Visible Light Communication "At the heart of this technology is a new generation of high brightness light-emitting diodes",

Hass says. Hass also included the transmission process as "Very simply, if the LED is on, a digital 1 is transmitted, if it's off 0 is transmitted. They can be switched on and off very quickly, which gives nice opportunities for transmitted data." The data to be transmitted is first encoded in to the light through varying the rate of flickering that generates different strings of 1's and 0's. Generally the modulation rate of LED is so rapid that the human eye cannot detect the variation and the output is generated continuously.

Li-Fi was mainly designed to overcome the drawbacks of WiFi usage. White LED is expected to replace fluorescent and incandescent lights in future and can be regarded as text generation lighting source. LED can support high speed on and off which can help in acquiring high data rate. Since in case of Li-Fi system optical source of light been used as a data transfer medium it can be safely used at the places where radio waves can harm the environment. Hospitals and Aircraft that are prone to the interference made by the radio waves can now make use of LED light as a safe mode of data transmission with no interference of waves. Unlike the Wi-Fi system, Li-Fi can be used in under water environment which increases the scope to be used in military operations.

\section{RELATED WORK}

- Notable work has been done by WANG Jia-yuan, ZOU and group mates in [1]. In the paper Experimental study on visible light communication based on LED paper they have talked about the data rate of LI-Fi i.e. $111.607 \mathrm{kbps}$ and communication distance i.e. $1.5 \mathrm{~m}$. Also it is known that Li-Fi is based on VLC through the paper.

- In 2012, the paper published by Jyoti Rani and teammates was $\mathrm{Li}-\mathrm{Fi}$ - The future technology in wireless communication [2]. Through this paper it is understood that transmission is done by taking fibre out of fibre optics and data is sent through LED light.

- In 2014, Navyatha's group published the paper Li-Fi-Led based alternatives [3]. Parallel data transmission is done by using any spectrum of light like red, green, blue.

- Raunak and group published the paper Li-Fi technology [4]. Due to the low cost nature of LEDs and lighting units, there are many opportunities to exploit from public internet access through street lamps to auto- piloted cars.

- In the paper Li-Fi: A Reconnaissance of future technology [5]. Li-Fi includes sub-gigabit, gigabit- class communication speeds for short, medium and long ranges.

- Another paper published in 2014 by was by Vitthal and group by name of Next of Wi-Fi - a future technology in Wireless Networking Li-fi using LED over Internet of Things [6]. Li-Fi idea is same as that of infrared remote controls but is more powerful hence called D-LIGHT.

- In 2015, Gagandeep Kaur Virk published the paper Li-fi: A New Communication Mechanism [7]. Li-Fi is more secure as light waves cannot penetrate through walls and cannot be intercepted by anyone outside the illumination of LED. 


\section{PROPOSED MODEL}

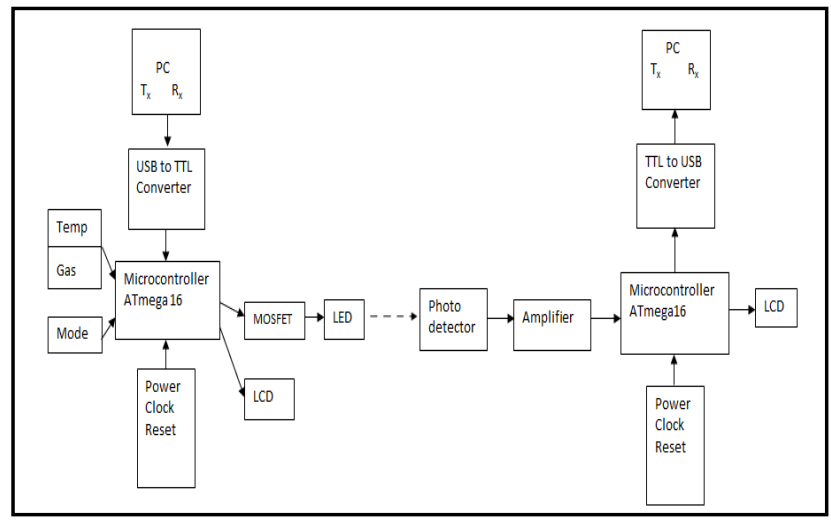

Figure 1: Block Diagram

\subsection{Transmitter Side}

To transmit data, an USB interface is used which is easy to connect to the computer; but USB level data cannot be given directly to light source so it needs to be converted it into serial data format as per the protocol. For this, a USB to TTL converter is being used.The data from the converter is given to microcontroller ATMega 16 and it converts the data to a particular format that it understands. To activate the microcontroller, 3 more components are used: power supply, clock and reset. The data which was given to microcontroller is an electronic signal pulse so it needs to be amplified. Hence, a MOSFET amplifier is used which amplifies the signal and transmits it to the photodiode through the LED light. Data transmission is done for not only computer data but also industrial data. For that, two sensors are placed in the transmitter side i.e. temperature sensor and gas sensor. Also, a mode selector is attached which helps to select whether computer data or industrial data is to be transmitted.

\subsection{Receiver Side}

The photodiode in the receiver side receives the data coming from LED and the signal is amplified. Then, it is given to microcontroller so that it is converted to a particular format and fed to TTL to USB converter. It is then seen in the computer.

\section{CIRCUIT DIAGRAM}

\subsection{Transmitter}

At the transmitter part, Microcontroller AT mega 16 is used at the center of the circuit, here AT stands for Atmel Company with mega series having 16 kilo bytes of flash memory which is used for programming. To start the microcontroller, power supply, clock and reset are used. The microcontroller consist of 40 pins, where power supply of $5 \mathrm{~V}$ is provided at pin 11 and 31 , pin 10 and 30 is allocated for ground. Crystal oscillator of $11.0592 \mathrm{MHz}$ is attached at pin 12 and 13 which is connected with two 22pf capacitor which is grounded which helps in activating the oscillator. This provides a clock source helps in executing the program. A reset setup is provided at pin 9 where a register of $1 \mathrm{~K}$ and capacitor of $1 \mu \mathrm{f}$ are connected which is called a power on reset as when the power is switched on the microcontroller sends a pulse which reset the controller and the program starts from 0th position of memory.

The controller consists of four ports that are PA, PB, PC and $\mathrm{PD}$ which are 8 pin bidirectional ports. In case of PD the first two pins are used for serial communication where the TX pin of USB to TTL convertor is connected to the RX pin of PD and the RX pin of convertor is connected to the TX of PD grounding the third pin this makes data to flow into the controller. To keep a check on the current status of the controller a LCD display of $16^{*} 2$ which symbolizes 16 character and 2 rows is connected to PC. At VCC pin of LCD power supply of $5 \mathrm{v}$ is provided, variable register of $10 \mathrm{k}$ is connected to the Contrast pin which varies the brightness of the LCD. LCD display consists of 8-bit data (D0-D7) bus having three control pins that are data/command, Read/write and enable and remaining D4, D5, D6, D7 are connected to PD so it is called as 4 bit mode. In 4-bit mode 4-bit data is send twice on joining $4+4=8$ bit data is finally displayed on LCD advantage of this is LCD can display the status by connecting to just one port.

Here, industrial application is also included for which sensors are added which is connected at PA. For Temperature sensing we have thermistor of $10 \mathrm{~K}$ NTC attached to PA, as the temperature changes resistance changes but the voltage remains the same thus connect a resistor to have potential divider and the junction output is given to the controller. Now for gas sensing, MQ6 identifies the flammable gases and a heater plate is connected to $5 \mathrm{v}$ supply and ground. The output of the sensing plate is given to the resistor which is connected to the controller. A switch is connected to PB for selecting computer data or sensor data to be transmitted. To send the data LED light module is connected which has multiple LED source that requires $12 \mathrm{v}$ supply, this LED requires high supply so for amplification purpose a MOSFET IRF280 is connected parallel to LED.

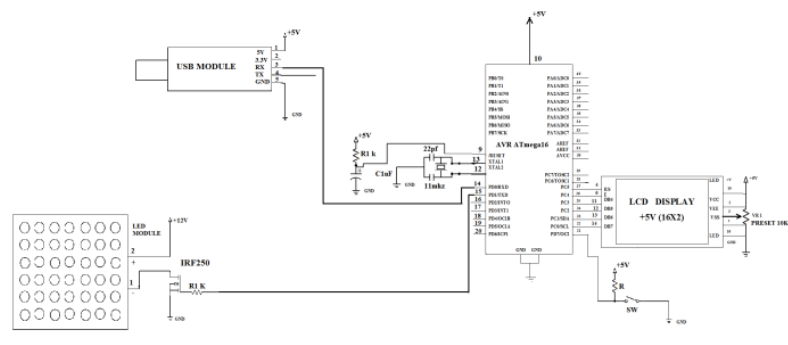

Figure 2: Transmitter's Circuit Diagram

\subsection{Receiver}

Photo diode is used on the receiver side for receiving the data. Since the signal of photo diode is quite weak a transistor is used to amplify the signal. The data is received and the status is displayed on the LCD. Here TTL to USB convertor is used which consists of Rx, Tx and ground pins. The Tx pin of the microcontroller is connected to the Rx pin of the TTL to USB convertor. Unlike transmitter section receiver side does not have any mode selection or temp and gas sensors connected to it. Main power supply is $230 \mathrm{~V}$. A step down transformer is used to step down to $12 \mathrm{~V}$ supply $1 \mathrm{amp}$. As this is AC, a bridge rectifier is used to rectify in order to convert it to DC. After it is rectified it is filtered and connected to transformer. The voltage from main power supply is stepped down then rectified and filtered. A voltage regulator i.e. 7805 is attached. Complete digital circuit requires $5 \mathrm{~V}$ power supply, therefore, $5 \mathrm{~V}$ ground is connected everywhere. 


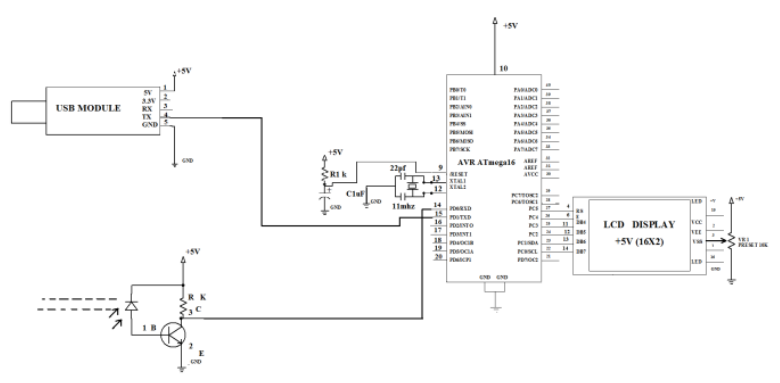

Figure 3: Receiver's Circuit Diagram

\section{RESULTS}

Around 115200 baud rate speed is achieved during transmission of data. Also, text messages, image files, audio files and video files are sent successfully using this technique.

\subsection{Sensor Mode}

In sensor mode, gas and temperature sensors are used. The temperature can be changed by touching the thermistor and the gas units too can be changed. Com 4 is the transmittor and Com 3 is the receiver. Whatever changes are made in the temperature are visible in the receiver part of the X-CTU software.

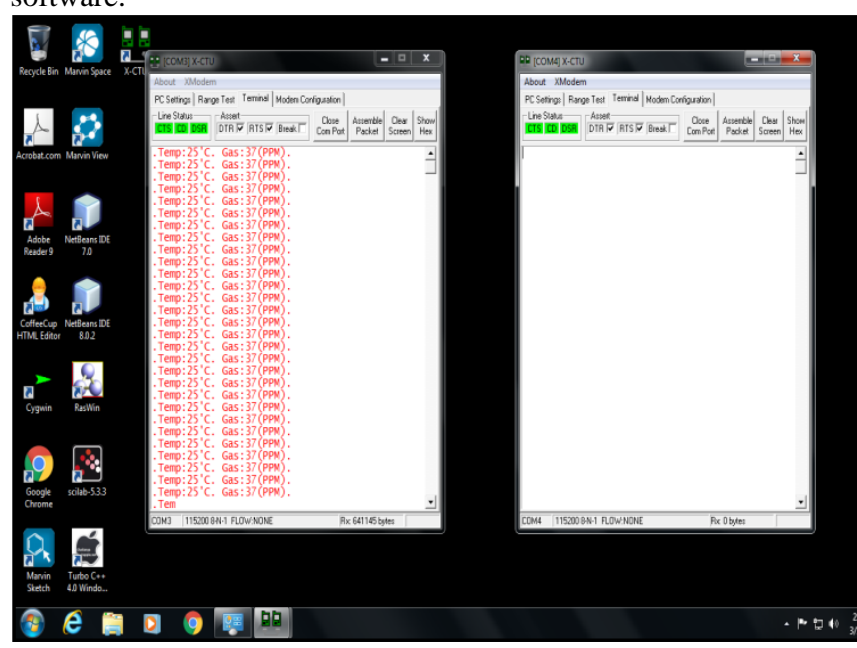

Figure 4: Sensor Mode (1)

On disturbing the transmission of the temperature and gas units, garbage values are obtained as shown below.

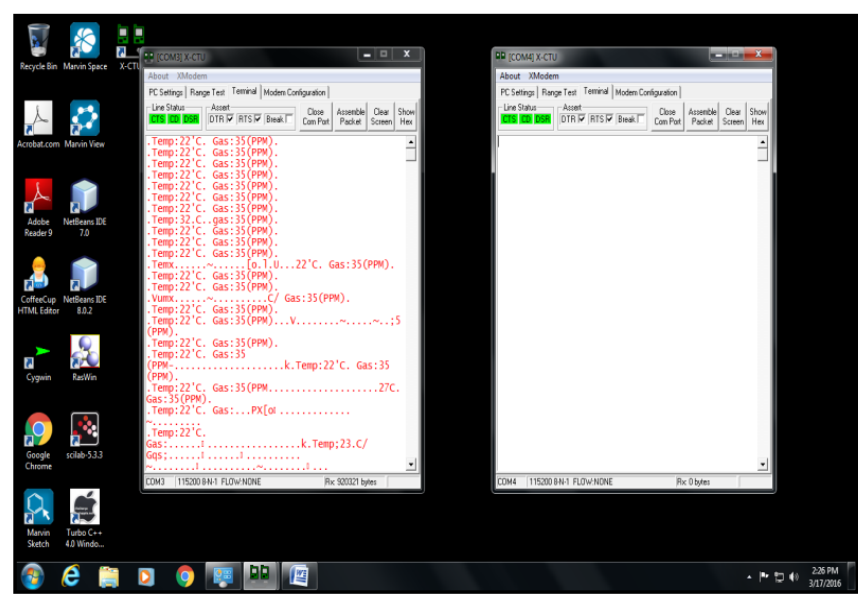

Figure 5: Sensor Mode (2)

\subsection{PC Mode}

In PC mode, text messages are sent from transmitter to the receiver. Here also Com 4 is the transmitter whereas Com 3 is the receiver. Whatever is typed in the transmitter section is displayed simultaneously in the receiver section.

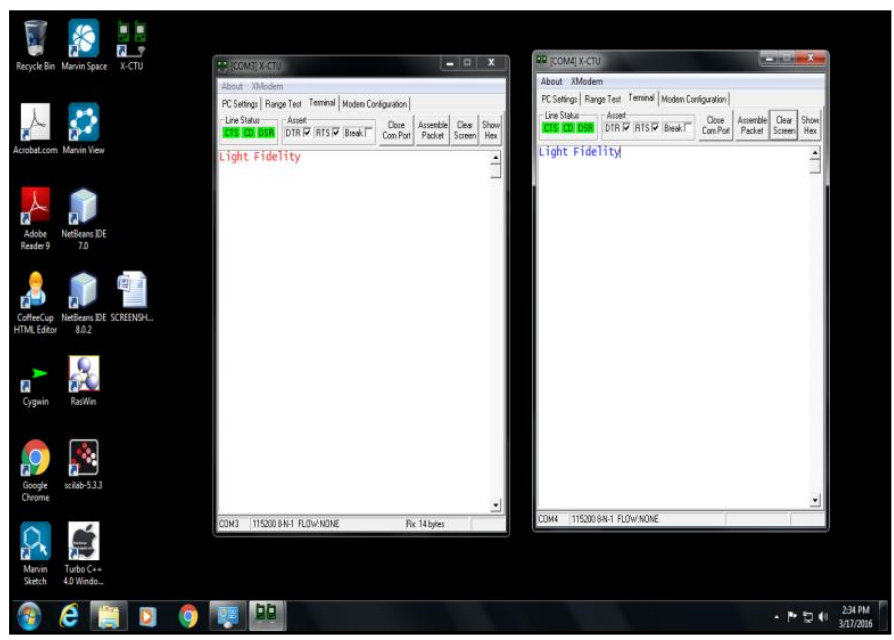

Figure 6: PC Mode (1)

On disrupting the flow of data, garbage value is obtained in the form of dots.

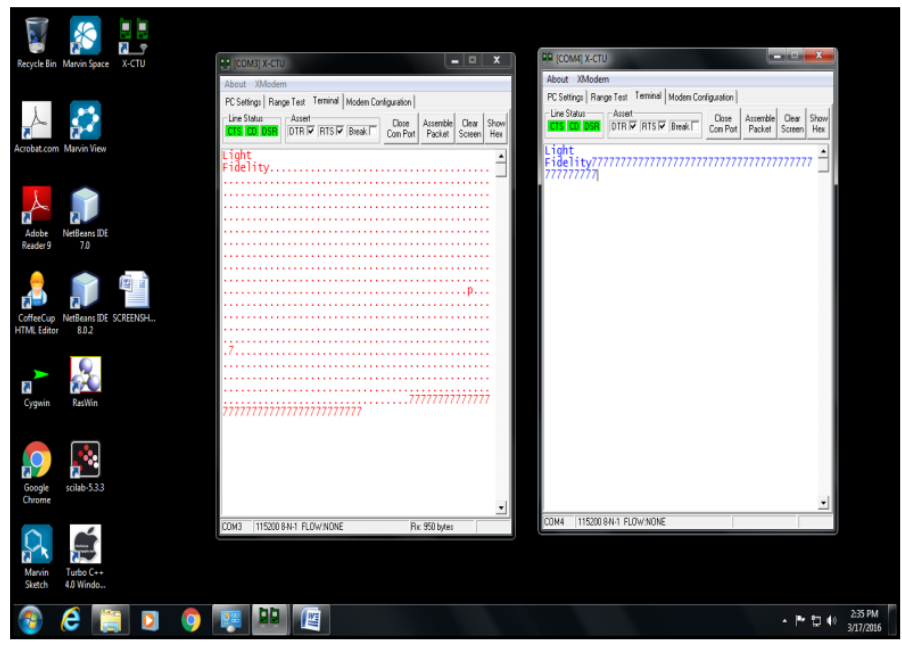

Figure 7: PC Mode (2)

\subsection{Image and Video Transmission}

When image is transmitted, File Send software is opened which is saved at C: on opening the File Send software first select the sender and the receiver and then change the settings i.e. label the dialogue box as the one for transmitter and the other one for receiver. After the settings are done the receiver is set to listen only then the transmitter starts sending the files. Similarly, video is also transmitted. 


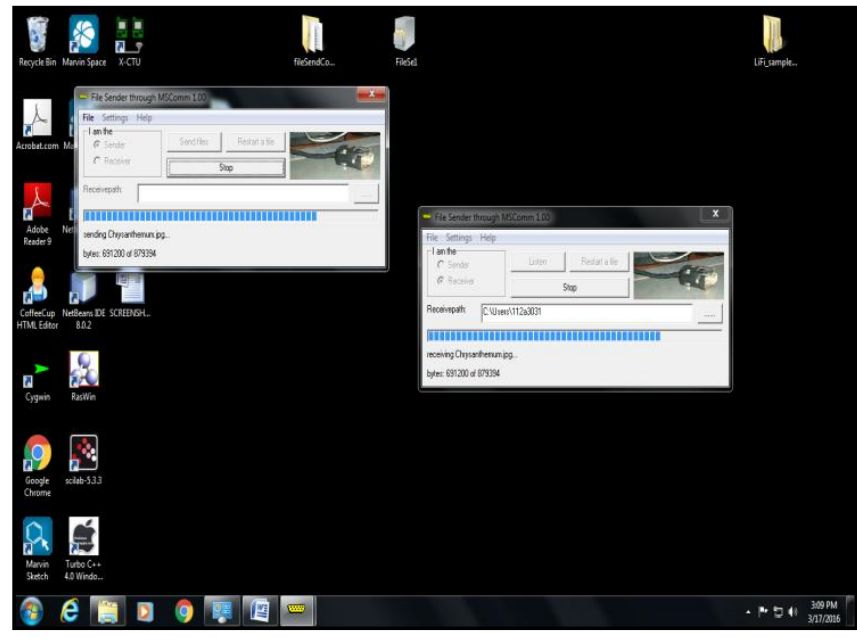

Figure 8: During transmission of image

Image Chrysanthemum is displayed in the set location (Figure 10).

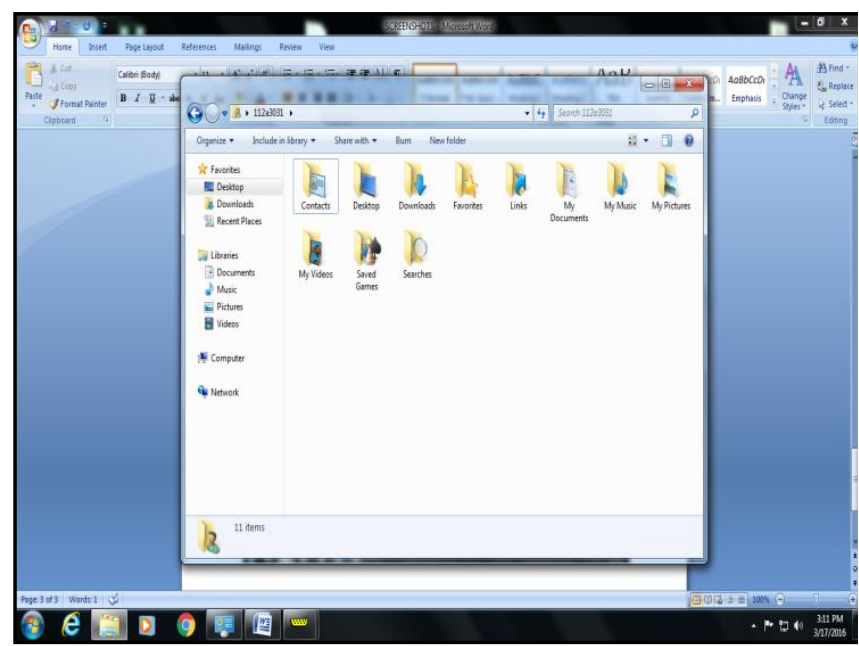

Figure 9: Before Transmission

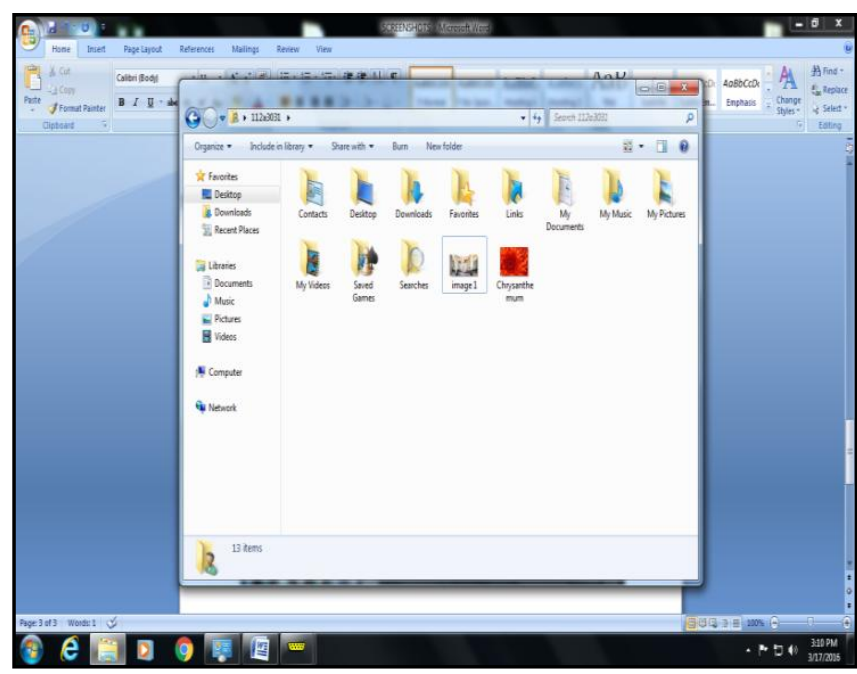

Figure 10: After Transmission

The above figures can be summarized as below:
Table 1: Analyzed Data

\begin{tabular}{|c|c|c|}
\hline $\begin{array}{c}\text { Transmission } \\
\text { Mode }\end{array}$ & $\begin{array}{c}\text { Without } \\
\text { Disturbance }\end{array}$ & $\begin{array}{c}\text { With } \\
\text { Disturbance }\end{array}$ \\
\hline Sensor Mode & $\begin{array}{c}\text { Gas and } \\
\text { temperature units } \\
\text { are displayed in a } \\
\text { sequence as } \\
\text { shown in figure 4 }\end{array}$ & $\begin{array}{c}\text { Gas and } \\
\text { temperature units } \\
\text { are displayed } \\
\text { along with } \\
\text { garbage values as } \\
\text { shown in figure 5 }\end{array}$ \\
\hline PC Mode & $\begin{array}{c}\text { Data is transmitted } \\
\text { properly as shown } \\
\text { in figure 6 }\end{array}$ & $\begin{array}{c}\text { Garbage values } \\
\text { are displayed in } \\
\text { the form of dots as } \\
\text { shown in figure 7 }\end{array}$ \\
\hline Image & $\begin{array}{c}\text { Image opens at } \\
\text { desired location } \\
\text { without any error } \\
\text { as shown in fig 10 }\end{array}$ & Image file gets \\
corrupted
\end{tabular}

Table 2: Comparison between $\mathrm{Li}-\mathrm{Fi}$ and Wi-Fi

\begin{tabular}{|c|c|c|}
\hline Parameter & Li-Fi & Wi-Fi \\
\hline Speed & High & High \\
\hline Range & Low & High \\
\hline Communication & $\begin{array}{c}\text { Based on Visible } \\
\text { Light } \\
\text { Communication }\end{array}$ & $\begin{array}{c}\text { Based on Radio } \\
\text { Frequency }\end{array}$ \\
\hline Data Density & High & Low \\
\hline Security & High & Medium \\
\hline $\begin{array}{l}\text { Ecological } \\
\text { Impact }\end{array}$ & Low & Medium \\
\hline $\begin{array}{c}\text { Transmit/ } \\
\text { Receive power }\end{array}$ & High & Medium \\
\hline $\begin{array}{l}\text { Infrastructure } \\
\text { Cost }\end{array}$ & Less & More \\
\hline Efficiency & $\begin{array}{c}\text { More. LEDs } \\
\text { consume less } \\
\text { energy and are } \\
\text { highly efficient }\end{array}$ & $\begin{array}{c}\text { Less. Radio Base } \\
\text { Stations consume } \\
\text { high amount of } \\
\text { energy }\end{array}$ \\
\hline Availability & $\begin{array}{l}\text { Anywhere. It can } \\
\text { be available in } \\
\text { airplanes, under } \\
\text { water with the } \\
\text { help of LED bulbs }\end{array}$ & $\begin{array}{c}\text { Limited because } \\
\text { of the harmful } \\
\text { effects }\end{array}$ \\
\hline
\end{tabular}




\section{CONCLUSION}

\subsection{Advantages}

- Li-Fi works like a wide-area Wi-Fi hotspot as the light waves do not penetrate through walls and hence it is very secure.

- It provides very low electromagnetic interference which allows connectivity in areas where $\mathrm{Wi}-\mathrm{Fi}$ is not allowed.

- Also, the equipment used in the project are very cheap so the overall cost is low.

\subsection{Applications}

- Since Li-Fi uses just light, it can be used safely in aircrafts and hospitals which are prone to interference from radio waves.

- Radio waves are quickly absorbed in water but light can penetrate large distances and headlamps are used for communicating under water.

- $\quad$ Li-Fi can be used in petroleum or chemical plants where other transmission or frequencies could be hazardous.

\section{ACKNOWLEDGMENTS}

Thanks to the guide Prof. K. Lakshmisudha who have contributed towards development of the project.

\section{REFERENCES}

[1] WANG Jia-yuan,ZOU Nian-yu,WANG Dong, "Experimental Study on visible light communication based on LED", www.sciencedirect.com/science/article/pii/S1005888511 604226

[2] Jyoti Rani, Prerna Chauhan, Ritika Tripathi, "Li-Fi (Light Fidelity)-The future technology In Wireless communication" , International Journal of Applied Engineering Research, ISSN 0973-4562 ,Vol.7, No.11 ,2012.

[3] N. Navyatha, T.M. Prathyusha, V. Roja, M. Mounika,"Li-Fi(Light Fidelity): LED based alternative",www.ijser.org/researchpaper\%5CLi-FiLight-fidelity-LED-Based-Alternative.pdf

[4] Rahul.R.Sharma, Raunak, Akshay Sanganal, "Li-Fi Technology", www.ijcta.com/documents/volumes/vol5issuel/ijcta2014 050121.pdf

[5] Dhakane Vikas Nivrutti, Ravi Ramchandra Nimbalkar ,Li-Fi: A Reconnaissance of future technology, http://www.ijarcsse.com/docs/papers/Volume_3/11_Nov ember2013/V3I11-0434.pdf

[6] Vitthal.S, Saptasagare: Next of Wi-Fi an future technology in wireless networking Li-fi using LED over internet of Things

[7] Gagandeep Kaur Virk,"Li-Fi : A new communication mechanism",

research.ijcaonline.org/volume118/number15/pxc390342 1.pdf

[8] Jay H. Bhut, Dharmrajsinh N. Parmar, Khushbu V. Mehta, "LI-FI Technology - A Visible Light Communication", https://www.ijedr.org/papers/IJEDRCP1401007.pdf

[9] Sinku U. Gupta, "Research on Li-Fi Technology\& Comparison of "Ri-Fi/Wi-Fi", http://www.lemarson.com/public/upload/ressource/filena me/Li-Fi_Gupta.pdf

[10] Giriraj KR. Patidar, "LI-FI TECHNOLOGY IN WIRELESS COMMUNICATION", http://www.ijset.in/wpcontent/uploads/2014/12/122014.1389_LiFi_126-130.pdf

[11] M. A. Hadi, "Wireless Communication tends to Smart Technology Li-Fi and its comparison with Wi-Fi", http://www.ajer.org/papers/v5(05)/F0505040047.pdf

[12] Smita Pawar, Tyran Kinny, Franklin Puthuva, Ashore Komban, Dipti Belekar, "DATA COMMUNICATION USING VISIBLE LIGHT", http://www.giapjournals.org/uploads/2/6/6/2/26621256/d ata_communication_using_visible_light.pdf

[13] Bhavya R., Lokesh M. R, "A Survey on Li-Fi Technology", http://www.aijet.in/v3/1601003.pdf

[14] Deepali Bajaj, Isha Mangal, Asha Yadav, "Towards an understanding of Li-Fi: Next generation Visible Light Communication http://www.ijecs.in/issue/v4-i4/101\%20ijecs.pdf 